\title{
The Hydrodynamics of High Diving ${ }^{\dagger}$
}

\author{
Thibault Guillet ${ }^{1}$, Mélanie Mouchet ${ }^{1}$, Jérémy Belayachi ${ }^{1}$, Sarah Fay ${ }^{2}$, David Colturi ${ }^{3}$, \\ Per Lundstam ${ }^{3}$, Peko Hosoi ${ }^{2}$, Christophe Clanet ${ }^{1}$ and Caroline Cohen ${ }^{1, *}$ \\ 1 LadHyX, École polytechnique, 91128 Palaiseau, France; thibault.guillet@polytechnique.edu (T.G.); \\ mouchet@polytechnique.edu (M.M.); belayachi@polytechnique.edu (J.B.); clanet@polytechnique.edu (C.C.) \\ 2 Department of Mechanical Engineering, MIT, Cambridge, MA 02139, USA; scfay@mit.edu (S.F.); \\ peko@mit.edu (P.H.) \\ 3 RedBull, Santa Monica, CA 90404, USA; dcolturi@gmail.com (D.C.); per.lundstam@redbull.com (P.L.) \\ * Correspondence: cohen@ladhyx.polytechnique.fr; Tel.: +33-169335260 \\ + Presented at the 13th Conference of the International Sports Engineering Association, Online, 22-26 June 2020.
}

Published: 15 June 2020

\begin{abstract}
Diving consists of jumping into water from a platform, usually while performing acrobatics. During high diving competitions, the initial height reaches $27 \mathrm{~m}$. From this height, the crossing of the water surface occurs at $85 \mathrm{~km} / \mathrm{h}$, and as such it is very technical to avoid injuries. Major risks occur due to the violent impact at the water entry and the formation and collapse of the air cavity around the diver. In this study, we investigate experimentally the dynamics of the jumper underwater and the hydrodynamic causes of injuries in high dives by monitoring dives from different heights with high-speed cameras and accelerometers in order to understand the physics underlying diving.
\end{abstract}

Keywords: sports hydrodynamics; cliff diving; injuries; water entry; air cavity

\section{Introduction}

Diving consists of falling or jumping through air into water from a platform, usually while performing acrobatics. During high diving competitions, the initial height reaches $27 \mathrm{~m}$. From this height, the crossing of the water surface occurs at $85 \mathrm{~km} / \mathrm{h}$ and as such it is very technical to avoid injuries [1-5]. Major risks occur from the violent impact at the water entry and the formation and collapse of the air cavity around the diver, if his body is not perfectly vertical and stiffened [6,7]. For Olympic competitions, the initial heights are 1,3 or $10 \mathrm{~m}$. In these cases, the athletes can enter the water with their arms forwards [8]. In a high dive, athletes always enter feet first and are limited to less than ten dives a day to avoid injury. It is well known among them that a transition occurs at 14 $\mathrm{m}$, the height at which diving arms first becomes dangerous. Finally, the maximal height from which an athlete can jump into water without injury (feet first) is $58 \mathrm{~m}$.

In this study, we investigate experimentally the dynamics of the jumper underwater and the hydrodynamic causes of injuries in a high dive, by monitoring one male top-level cliff diver, jumping from different heights during his habitual training with high-speed cameras both in air and underwater and with an accelerometer fixed on his body. The final goal of this work is to better understand the physics underlying high cliff diving as inspired by the studies of the water entry of projectiles [9-11] and to be able to protect divers by monitoring their dives with embedded sensors or by designing safety gear. 


\section{Materials and Methods}

Several dives were executed by a professional diver $(1 \mathrm{~m} 72,70 \mathrm{~kg})$ during a summer training session in the RedBull park Area 47 in Austria. The diving platform has several stages ranging from 0.7 to $27 \mathrm{~m}$, which enables us to vary the speed impact at the water entry. Each dive was monitored with an accelerometer acquired at $100 \mathrm{~Hz}$ inserted in the diver's suit, on his chest (Figure 1a top) and two high-speed cameras (1000 fps): one recording the trajectory of the diver in air and the other immersed 3-m deep in a waterproof box (Figure 1a bottom), designed to observe the diver's trajectory underwater.

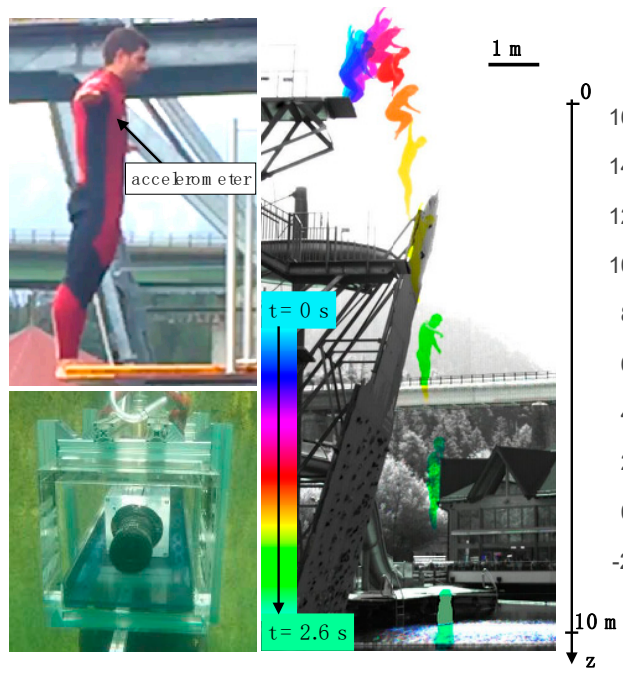

(a)

(b)

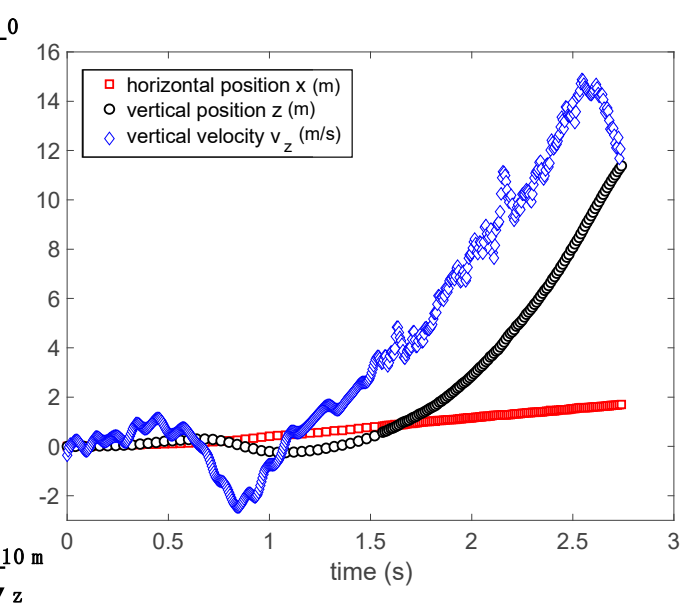

(c)

Figure 1. (a) Experimental setup: an accelerometer is inserted in the suit of the diver (top), and a highspeed camera is immersed at 3-m deep in a waterproof box to record the underwater trajectory (bottom); (b) Colored chronophotograph of the jump: time elapses from light blue to green, with a timestep of $0.2 \mathrm{~s}$ between two images; (c) Evolution of the horizontal (x) and vertical downward (z) positions and vertical velocity $\left(\mathrm{V}_{\mathrm{z}}\right)$ in function of time. The origins of time and positions are taken at the beginning of the jump for the aerial part.

\section{Results}

\subsection{Trajectory of the Diver in Air-High-Speed Camera 1}

Figure $1 \mathrm{~b}$ exhibits a colored chronophotograph of a $10 \mathrm{~m}$-high dive: the first phase of the jump is the impulsion. The diver bends his legs (his center of mass moves down of approximately $40 \mathrm{~cm}$ ) and pushes on the platform in order to jump as high as possible (approximately $70 \mathrm{~cm}$ upwards) and to move forward, far from the platform. He first rolls into a ball and then stretch his body in order to impact water as rigid, slender and aligned as possible. The whole jump lasts $2.55 \mathrm{~s}$ and the fall from the take-off until the water entry lasts $1.75 \mathrm{~s}$. The trajectory of the center of mass is extracted from the video and enables us to plot the time-evolution of the vertical $(\mathrm{z})$ and horizontal $(\mathrm{x})$ positions and to calculate the vertical velocity $(\mathrm{vz})$. After the end of the impulsion $(\mathrm{t}=0.8 \mathrm{~s})$, one observes a quadratic evolution of the vertical position and, after reaching the maximal height $(t=1.1 \mathrm{~s})$, a linear increase of the vertical speed during the fall, with confirms the ballistic movement of the center of mass.

\subsection{Trajectory of the Diver in Water-High-Speed Camera 2}

The following of the trajectory begins when the feet impacts the water. It can be observed in Figure 2 that the diver's body goes on downwards but decelerates. The forces undergone by the diver at the origin of both the deceleration and risks of injury are discussed in Section 4.3. On the chronophotograph of the water entry (Figure 2a), one notes the presence of a cavity due to the air entrainment by the feet crossing the air-water interface, which then collapse onto the diver. 


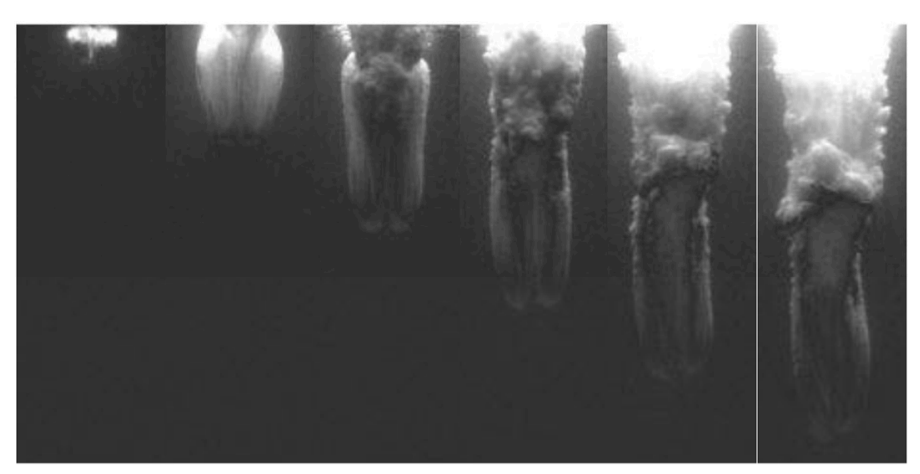

(a)

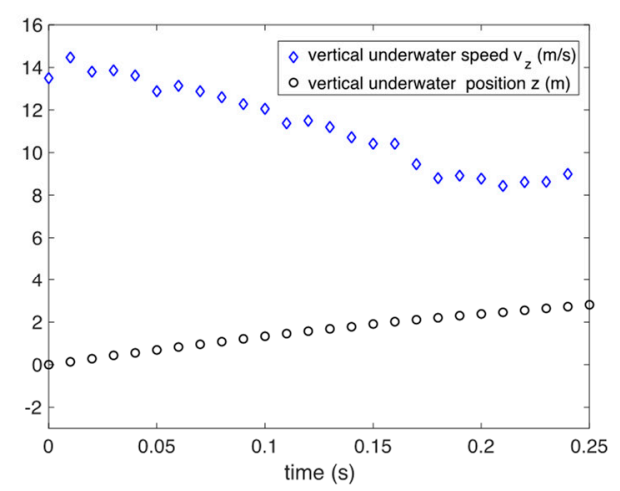

(b)

Figure 2. Trajectory of the diver's center of mass in water for the same 10-m high dive: (a) Chronophotograph of the water entry. The timestep is $40 \mathrm{~ms}$. (b) Time evolution of the downward vertical position $(\mathrm{z})$ and speed $\left(\mathrm{V}_{\mathrm{z}}\right)$ underwater. The origins of time and position are taken upon the feet impact on water for the underwater phase.

\subsection{Acceleration of the Diver}

Figure 3 shows the accelerometer data obtained during the same 10-m high dive. Again, one observes three phases. First the diver bends his legs: the vertical acceleration $\left(-\mathrm{az}_{\mathrm{z}}\right)$, becomes negative and then increases and becomes positive again when he pushes on the platform, and takes off. After, he reaches the maximal height and is only submitted to the gravity field $(\mathrm{g})$ modulo fluctuation, probably due to the movements and deformation of the body. After $2.55 \mathrm{~s}$, the feet impact the water surface and create an intense peak $(6.9 \mathrm{~g})$ of acceleration corresponding to the upwards force suffered by the diver. This peak is followed by a series of other peaks and oscillations corresponding to the collapse of the air cavity surrounding the diver. The goal of the following section is to understand the observed dynamics and deduce the forces suffered by the diver during the water entry and the injury risks.

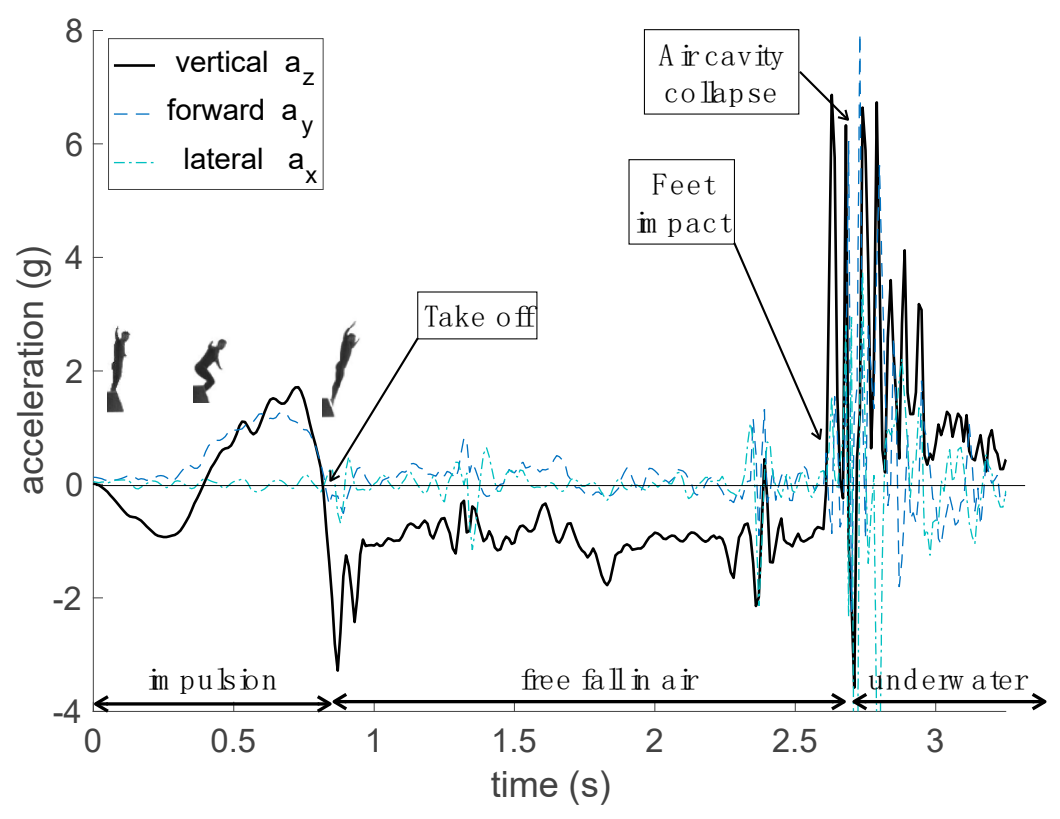

Figure 3. Time evolution of the acceleration of the diver during the whole dive (aerial and underwater $)$ in the three direction: vertical $\left(-\mathrm{a}_{\mathrm{z}}\right)$, forward $\left(\mathrm{a}_{\mathrm{x}}\right)$ and lateral $\left(\mathrm{a}_{\mathrm{y}}\right)$. Time origin is taken at the beginning of the impulsion. The measured vertical acceleration is orientated upwards (gravity $g$ downwards). 


\section{Discussion}

\subsection{Impulsion}

It is interesting to characterize precisely the impulsion of the diver as the maximal height reached during this phase will set the velocity at the water entry [8]. This maximal height reached can be first accessed thanks to the video recording of the trajectory in air. One measure is that the diver lowers his center of mass of approximately $z_{0}-z_{\text {bent }}=40 \mathrm{~cm}$, when he bends his legs and then rises it up of $z_{\text {max }}-z_{\text {bent }}=70 \mathrm{~cm}$, finally increasing the maximal height of $z_{\text {max }}-z_{0}=30 \mathrm{~cm}$ from the initial position. To recover these results with only the accelerometer data, one can consider the vertical acceleration occurring during the impulsion as a linear increase with a slope equal to $\alpha=5.9 \mathrm{~g}$ and during $t_{a}=0.42 \mathrm{~s}$. By integrating this acceleration signal $-a_{z}=\alpha t$, one gets a variation of vertical position $z_{0}-z_{\text {bent }}=\alpha t_{a}^{3} / 6$ and finally a value of $z_{\max }-z_{\text {bent }}=71 \mathrm{~cm}$, which is in good agreement with the previous observations.

\subsection{Free Fall in Air}

From this initial height $H_{\max }=H+z_{\max }-z_{0}$, one would like to determine the velocity reached by the diver when he reaches the water surface. As suggested by the measurements exposed in Section 3.1, the trajectory in air is ballistics. Indeed, one can evaluate the typical drag force exerted on the diver, which is opposed to his displacement $F_{D}=\frac{1}{2} \rho_{\text {air }} S C_{D} v_{z}^{2}=4 \mathrm{~N}$ (where $\rho_{\text {air }}=$ $1.2 \mathrm{~kg} / \mathrm{m}^{3}$ is the air density, $S \approx 0.16 \mathrm{~m}^{2}$, the cross section of the diver and $C_{D} \approx 0.2$, his drag coefficient) and compare it to the weight of the diver $M g=686 \mathrm{~N}$. One can thus neglect the aerodynamical effect during the dive, and finally obtain a free fall. The velocity reached just before hitting the water $\left(U_{0}\right)$ is thus immediately given by the maximal height reached by the diver: $U_{0}=$ $\left(2 g H_{\max }\right)^{1 / 2}$. Figure 4 shows the experimental velocities measured at the water entry for several dives from different platform heights $\mathrm{H}$, ranging from 0.7 to $27 \mathrm{~m}$ and the predicted ones, taking into account the initial rise of $30 \mathrm{~cm}$ due to the impulsion.

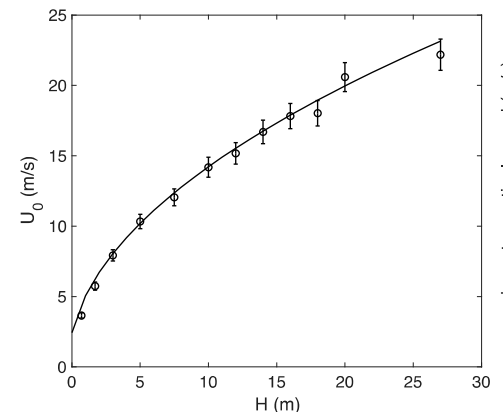

(a)

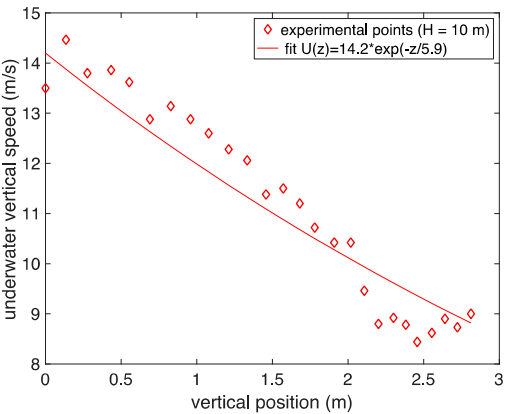

(b)

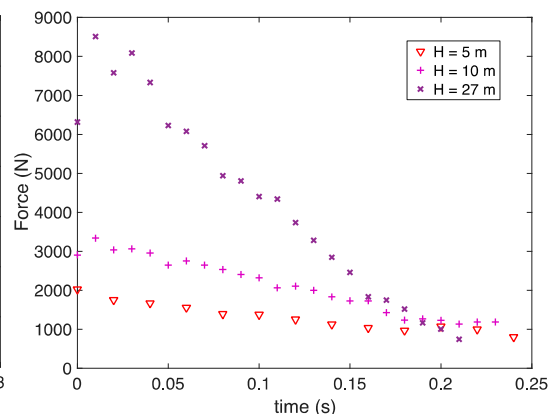

(c)

Figure 4. (a) Velocity of the diver at the water entry. Open circles show the experimental values for several dives from different platform heights $\mathrm{H}$, ranging from 0.7 to $27 \mathrm{~m}$ and the solid line gives the predicted value $U_{0}=\left(2 g H_{\max }\right)^{1 / 2}$. (b) Evolution of the underwater speed (U) with the vertical position (z). Red diamonds show experimental points and the line represents the fit of the theoretical expression given by Equation (1). (c) Force undergone by the diver underwater in function of time, for three different initial heights $\mathrm{H}=5,10$ and $27 \mathrm{~m}$, calculated from Equation (2).

\subsection{Water Entry}

Knowing the initial velocity at the water entry, one has now to understand the dynamics of the diver underwater [11]. The forces undergone by the diver are:

- the weight, $M g=0.7 \mathrm{kN}$

- $\quad$ the buoyancy, $\rho_{\text {water }} V_{d} g \approx 0.7 \mathrm{kN}$, with $\rho_{\text {water }}=\frac{997 \mathrm{~kg}}{\mathrm{~m}^{3}}$ and $V_{d}=\frac{\mathrm{M}}{\rho_{\text {body }}}$ and $\frac{\rho_{\text {body }}}{\rho_{\text {water }}}=0.9$.

- the hydrodynamic drag $F_{D}=\frac{1}{2} \rho_{\text {water }} S C_{D} \mathrm{U}^{2} \approx 3.2 \mathrm{kN}$ 
- the added mass force $M_{a} \frac{d U}{d t}$, corresponding to the fact that the diver has to accelerate forward a mass of water to go further. The added mass expression is given by the formula for a discus $M_{a}=\rho_{\text {water }} D^{3} / 6 \approx 21 \mathrm{~kg}$, which is approximately a third of the diver's mass.

These orders of magnitude allow us to neglect the weight and buoyancy (acting in reverse directions and approximately equals, once the full body is immersed) to focus on the dominant drag and added mass effects. The equation of motion of the diver can then be written as follows:

$$
\left(M+M_{a}\right) \frac{d U}{d t}=-\frac{1}{2} \rho_{\text {water }} S C_{D} U^{2}
$$

and rewritten in function of $z: \frac{d\left(U^{2}\right)}{d z}=-\frac{2 U^{2}}{L}$, and easily solved as: $U(z)=U_{0} \exp (-z / L)$, with $L=$ $\frac{2\left(M+M_{a}\right)}{\rho_{\text {water }} S C_{D}} \approx 6 \mathrm{~m}$ a characteristic length, which represents the typical deceleration distance. Figure $4 \mathrm{~b}$ shows a good agreement between the experimental evolution of the underwater velocity with the position and the fit of the exponential prediction for an initial speed $U_{0}=14.2 \mathrm{~m} / \mathrm{s}$ and a characteristic length $L=5.9 \mathrm{~m}$. With a qualitative validation of the equations used to describe the dynamics of the diver, we can use it to predict the force undergone by the diver when he enters into water [12], which opposes his motion:

$$
F=M_{a} \frac{d U}{d t}+\frac{1}{2} \rho_{\text {water }} S C_{D} U^{2}
$$

This force is plotted in function of time for three different initial dive heights on Figure 4c. One can note that the maximal force $\left(F_{\max }\right)$ occurs at the beginning, when the feet first hit the interface, and increases for a greater initial speed: $F_{\max } \approx 2,3$ and $8.5 \mathrm{kN}$ respectively for $\mathrm{H}=5,10$ and $27 \mathrm{~m}$. With such impact forces, one can easily understand that a high dive $(\mathrm{H}=27 \mathrm{~m})$ can turn very dangerous if the impact is not well prepared.

\subsection{Air Cavity Underwater}

In addition to the impact force felt by the diver, another risk of pain comes from the collapse of the air cavity formed when the diver crosses the interface. Accelerometer data show that high accelerations occur when the cavity collapses and during experiments, and the diver complained about a neck pain during the water entry of a $27-\mathrm{m}$ high dive. Figure 5 shows air cavities just at the moment of collapse for various initial dive heights from 1.7 to $27 \mathrm{~m}$. One can see that the size (both length and radius) of the cavity increases with the initial height and at $27 \mathrm{~m}$, the cavity collapses on the diver's neck. The collapse of these cavities has to be further investigated by equipping divers with pressure sensors and understanding the shapes of the collapsing cavities observed [13].
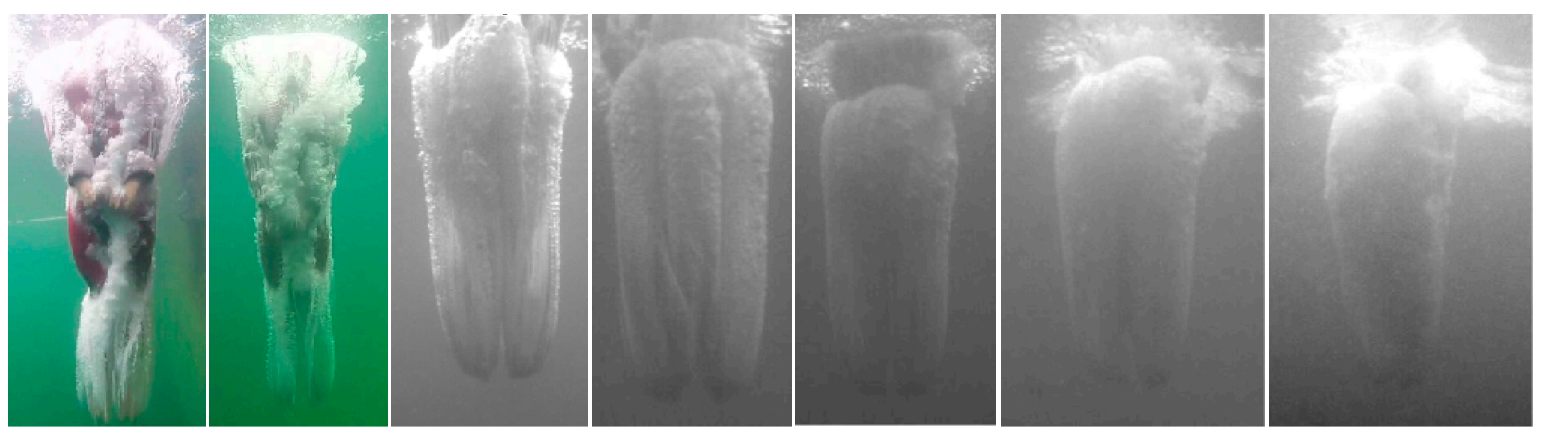

Figure 5. Pictures of air cavities taken at the moment of collapse for dives from several initial heights: $1.7,3,5,10,14,20$ and $27 \mathrm{~m}$ (from left to right). 


\section{Conclusions}

In this study, we focused on the dynamics of the high dive and its three phases: impulsion, free fall in air and underwater deceleration. By analyzing experimental data from two high speed cameras (in air and in water) and one accelerometer, we managed to predict the value of the force suffered by the diver when he impacts the water surface, namely the values of hydrodynamic drag and added mass effects. The perspectives of this study are to design embedded sensors able to automatically provide these forces to the divers in order to monitor their training, provide understanding around the risks they run and prepare them through appropriate muscular training.

Acknowledgments: The authors thank the École polytechnique for its funding and support of the Sports Physics initiative developed in LadHyX.

Conflicts of Interest: The authors declare no conflict of interest. The funding sponsors had no role in the design of the study; in the collection, analyses, or interpretation of data; in the writing of the manuscript, and in the decision to publish the results.

\section{References}

1. McElhaney, J.; Snyder, R.G.; States, J.D.; Gabrielsen, M.A. Biomechanical Analysis of Swimming Pool Neck Injuries. SAE Trans. 1979, 88, 494-500.

2. Napolitano, S. Cliff diving: Water impact and video-analysis. J. Phys. Educ. Sport 2014, 14, 93.

3. Ernstbrunner, L.; Runer, A.; Siegert, P.; Ernstbrunner, M.; Becker, J.; Freude, T.; Moroder, P. A prospective analysis of injury rates, patterns and causes in Cliff and Splash Diving. Injury 2017, 48, 2125-2131.

4. Rubin, B.D. The basics of competitive diving and its injuries. Clin. Sports Med. 1999, 18, 293-303.

5. Mountjoy, M. Injuries and medical issues in synchronized Olympic sports. Curr. Sports Med. Rep. 2009, 8, 255-261.

6. Chang, B.; Croson, M.; Straker, L.; Gart, S.; Dove, C.; Gerwin, J.; Jung, S. How seabirds plunge-dive without injuries. Proc. Natl. Acad. Sci. USA 2016, 113, 12006-12011.

7. Guillet, T.; Coux, M.; Quéré, D.; Clanet, C. Path instabilities of streamlined bodies. J. Fluid Mech. 2019, 864, 286-302.

8. Miller, D.I. Springboard and platform diving. Biomech. Sport 2000, 326. doi: 10.1002/9780470693797.ch16

9. Truscott, T.T.; Epps, B.P.; Belden, J. Water entry of projectiles. Annu. Rev. Fluid Mech. 2014, 46, 355-378.

10. Bodily, K.G.; Carlson, S.J.; Truscott, T.T. The water entry of slender axisymmetric bodies. Phys. Fluids 2014, 26,072108 .

11. Cohen, C.; Darbois-Texier, B.; Dupeux, G.; Brunel, E.; Quéré, D.; Clanet, C. The aerodynamic wall. Proc. R. Soc. A Math. Phys. Eng. Sci. 2014, 470, 20130497.

12. Truscott, T.T.; Epps, B.P.; Techet, A.H. Unsteady forces on spheres during free-surface water entry. J. Fluid Mech. 2012, 704, 173-210.

13. Duclaux, V.; Caillé, F.; Duez, C.; Ybert, C.; Bocquet, L.; Clanet, C. Dynamics of transient cavities. J. Fluid Mech. 2007, 591, 1-19.

(C) 2020 by the authors. Licensee MDPI, Basel, Switzerland. This article is an open access article distributed under the terms and conditions of the Creative Commons Attribution (CC BY) license (http://creativecommons.org/licenses/by/4.0/). 